\title{
Sodium-dodecyl-sulphate-assisted synthesis of Ni nanoparticles: electrochemical properties
}

\author{
RAZIYEH AKBARZADEH and HOSSEIN DEHGHANI* \\ Department of Inorganic Chemistry, Faculty of Chemistry, University of Kashan, Kashan, Iran \\ *Author for correspondence (dehghani@kashanu.ac.ir)
}

MS received 9 September 2016; accepted 3 April 2017; published online 20 November 2017

\begin{abstract}
Stabilized nickel nanoparticles (SNNPs) were prepared using Ni(acac) $)_{2}$ (acac = acetylacetonate) via a simple solvothermal method. The synthesis of the nickel nanoparticles was performed in the presence of sodium dodecyl sulphate (SDS) of different concentrations (mole ratios of SDS: $\mathrm{Ni}(\mathrm{acac})_{2}=1: 1,2: 1$ and 4:1), as the stabilizer, in order to appraise their influence on the morphology, size, dispersion, magnetic properties and electrochemical activity of the nickel nanoparticles. The synthesized products have been characterized by powder X-ray diffraction, scanning electron microscopy, transmission electron microscopy, Fourier transform infrared spectra, energy-dispersive X-ray spectroscopy, vibrating sample magnetometry and electrochemical studies. It is noteworthy that the average particles size of the SNNPs has been reduced by increasing the SDS concentration, while at high concentration (mole ratio of SDS:Ni(acac) $2=4: 1$ ), the small particles tend to coalesce and create a big one. The stabilized Ni nanoparticles could be used as electrode materials for hydrogen evolution in alkaline medium. The electrochemical measurements demonstrated that the higher conductivity and lower value of faraday resistance of the as-prepared samples were when the mole ratio of SDS: Ni(acac $)_{2}$ was 2:1.
\end{abstract}

Keywords. Nickel; SNNPs; stabilizer; SDS; electrochemical; nanoparticles.

\section{Introduction}

Over the last decades, synthesis of magnetic metallic nanomaterials with different sizes and shapes has received considerable attention due to their technological interest [1-5]. Nickel nanoparticles have become one of the attractive metallic nanomaterials, which are expected to exhibit interesting magnetic and electrochemical properties [6,7]. In recent years, nickel nanoparticles below $100 \mathrm{~nm}$ have been widely investigated due to their promising applications in the fields of chemical catalysis, rechargeable batteries, catalysis superconductors, magnetic recording media, optical, mechanical and electronic devices and so on [8,9]. Nickel and its alloys are among the new materials that show a high original electrocatalytic activity towards the hydrogen evolution reaction (HER) [10-12]. Various shapes of nickel nanocrystals, such as nanospheres, nanorods, nanobelts, nanotubes, hollow spheres and nanoprisms, have been successfully synthesized by different methods $[13,14]$. The synthesis of pure nickel nanostructured materials is comparatively difficult because they are easily oxidized. Metallic nickel nanoparticles have generally been produced by many physical and chemical methods such as electrochemical deposition, sol-gel technique, decomposition of organometallic precursors, polyol process, chemical vapour deposition (CVD), chemical reduction methods and microwave-assisted synthesis [15-19]. Among the various synthesis approaches, chemical reduction method using strong reduction agents is facile and suitable route for the synthesis of ferromagnetic nanoparticles. The widely used experimental method is based on the selection of stabilizers or surfactants for synthesis of metallic nickel nanostructures because depending on the functional groups on the stabilizers, they can interact with the surface of the particles and then permit the growth of the particles in a favourable direction [12]. Additionally, the stability of the nanostructures is normally achieved using different protective molecules (surfactants) to avoid their aggregation. Couto et al [20] prepared nickel nanoparticles $(3.7 \mathrm{~nm})$ via hydrothermal method with hexadecylamine (HDA) as both solvent and protective agent. Hou et al [21] employed HDA-trioctylphosphineoxide (TOPO)-oleic acid as a stabilizer to synthesize nickel nanostructures. Couto et al [20] modified the polyol process using polyvinylpyrolidone (PVP) as a surfactant. Crespo et al [12] used two different stabilizers, 1,3-diaminopropane (DAP) or anthranilic acid (AA), to generate stabilized Ni nanoparticles. Sodium dodecyl benzenesulphate (SDBS) was employed to produce nickel nanobelts [22]. Chou and Huang [23] synthesized nano-sized nickel colloids using PVP or poly(acrylic acid) PAA as polymeric dispersants in the presence of palladium chloride as a nucleation agent. Elsewhere, the solution reduction of $\mathrm{NiCl}_{2} \cdot 6 \mathrm{H}_{2} \mathrm{O}$ by hydrazine monohydrate using PVP was employed to produce nickel nanowires [24]. Recently, Sarkar et al [25] synthesized nickel nanostructures via reduction of nickel chloride in hydrazine monohydrate in the presence of various surfactants such polyethylene glycol $p$-(1,1,3,3-tetramethylbutyl)-phenyl ether (TX-100), 
cetyltrimethylammonium bromide (CTAB) and SDS in which they observed the formation of long nanowires for TX-100 and CTAB, but for SDS, no such nanowires were formed and only irregular spherical nanoparticles resulted [25]. In this paper, we report a stabilizer-assisted hydrothermal reduction method for synthesis of stabilized nickel nanoparticles (SNNPs) with high yield. The significant role of SDS as a stabilizing agent on the shape and size of NPs is demonstrated. Also, we report that the use of different concentrations of SDS not only affects size, morphology and dispersion but also influences the electrochemical activity of the nickel NPs. SNNPs were produced by reduction using appropriate amounts of $\mathrm{Ni}(\mathrm{acac})_{2}$ in N,N-dimethylformamide (DMF) solvent at various mole ratios of $\mathrm{SDS}: \mathrm{Ni}(\mathrm{acac})_{2}$ and relatively low temperature $\left(150^{\circ} \mathrm{C}\right)$.

\section{Experimental}

\subsection{Materials and physical measurements}

All reagents used in our experiments were of analytical grade and used without further purification. Nickel(II) acetylacetonate $\left(\mathrm{Ni}(\mathrm{acac})_{2}\right)$, sodium dodecyl sulphate (SDS) and DMF were procured from Merck. Absolute ethanol, deionized water and hexane were used during the preparation of powders. A Philips XL-30ESEM scanning electron microscope (SEM) equipped with an energy-dispersive X-ray spectroscopy (EDS) device was used to determine the morphology and composition of nanostructures, respectively. The transmission electron microscopy (TEM) image was obtained by dispersing the sample in ethanol and placing a drop of dispersion on a carbon-coated copper grid in a Zeiss EM10C instrument operating at $80 \mathrm{kV}$. Powder X-ray diffraction (XRD) study of the nickel NPs was made on a Phillips X'Pert PRO equipped with a $\mathrm{Cu} \mathrm{K} \alpha$ source $(\lambda=1.54178 \AA$ ) having a scanning range of $0-80$ Bragg's angle. Fourier transform infrared (FT-IR) spectrum was recorded with a Magna 550 Nicolet instrument, in the spectral range between 4000 and $400 \mathrm{~cm}^{-1}$, using $\mathrm{KBr}$ pellets. Additionally, magnetic characterization of the nickel NPs was performed to measure their magnetic properties at room temperature using a BHV-55, Riken, Japan, vibrating sample magnetometer (VSM).

\subsection{Synthesis of Ni nanoparticles}

Practically, the present approach to the synthesis of nickel nanoparticles is similar to the process that was used in our previous work [26]. In a typical experimental procedure, a desired amount of $\mathrm{Ni}(\mathrm{acac})_{2}(0.46,0.70$ and $0.93 \mathrm{mmol})$ was dissolved in $15 \mathrm{ml}$ of DMF. Then an appropriate amount of SDS as the stabilizer, according to $1: 1,2: 1$ and $4: 1$ mole ratios of SDS to $\mathrm{Ni}$ (acac) $)_{2}$, was added slowly to them with vigorous stirring. The mixture solution was transferred into a 20 $\mathrm{ml}$ Teflon-lined stainless-steel autoclave. Hydrothermal treatments were carried out at $150^{\circ} \mathrm{C}$ for $24 \mathrm{~h}$. Then, the autoclave was permitted to cool down naturally and the product was centrifuged. The black solid was collected and washed with absolute ethanol, deionized water and hexane several times and dried in air at $60^{\circ} \mathrm{C}$ for $6 \mathrm{~h}$.

\subsection{Electrochemical measurements}

The working electrodes were prepared according to the following steps; $10 \mu \mathrm{l}$ of an ultrasonically re-dispersed assynthesized nanoparticles suspension containing a 4:1 ratio of graphite:metal powders in pure ethanol was dropped onto a glassy carbon electrode (2 $\mathrm{mm}$ diameter), which was polished smoothly and washed cleanly in advance. After drying in a high-purity argon flow at room temperature, the deposited layer was then covered with $10 \mu \mathrm{l}$ of diluted aqueous Nafion solution and then dried in argon flow. Electrochemical properties of as-obtained electrodes were evaluated by cyclic voltammetry (CV) and electrochemical impedance spectroscopy (EIS) with an Autolab potentiostat-galvanostat PGSTAT 35 (Eco Chemie, Utrecht, Netherlands), coupled to a personal computer. The real $\left(Z^{\prime}\right)$ and imaginary $\left(Z^{\prime \prime}\right)$ components of impedance spectra were analysed with NOVA 1.6 fitting program to estimate the parameters of the different resistances and capacitances. The electrochemical measurements were performed in a three-electrode cell with $1 \mathrm{M}$ $\mathrm{NaOH}$ electrolyte at room temperature. A platinum plate and $\mathrm{Ag}-\mathrm{AgCl}$ were used for a counter-electrode and a reference electrode, respectively.

\section{Results and discussion}

To promote the formation of nickel nanoparticles and in order to evaluate the effect of stabilizer on size, dispersion and adjusting the morphology of nanoparticles, the anionic stabilizer SDS was used at different mole ratios $(1: 1,2: 1$ and $4: 1$ of SDS:Ni(acac) $)_{2}$, which acts as a stabilizing agent on the facets of the first-formed nanoparticles. Figure 1a-c shows the XRD patterns of the nickel NPs prepared through the thermal reduction of $\mathrm{Ni}(\mathrm{acac})_{2}(0.46 \mathrm{mmol})$ in $\mathrm{DMF}$ solvent in the presence of different amounts of SDS. The diffraction peaks can be indexed well to face-centred cubic nickel (JCPDS 04-0850). Three characteristic peaks at 44.6, 51.9 and $76.5^{\circ}$, which can be indexed, respectively, to the (111), (200) and (220) diffractions of fcc nickel, were observed and no apparent impurity phase is observed. This indicated that the as-synthesized nanoparticles were pure nickel. It was noted that for the Ni samples prepared in SDS, the widths of diffraction peaks were large on increasing the SDS concentration, suggesting that the particle size becomes slightly smaller. Subsequently, nickel NPs synthesized using SDS of various concentrations revealed similarly resolved XRD patterns, attributable to the fcc phase of nickel. Further, it shows that the stabilizing agent does not introduce any phase change and there is no considerable change in the position of peaks. 

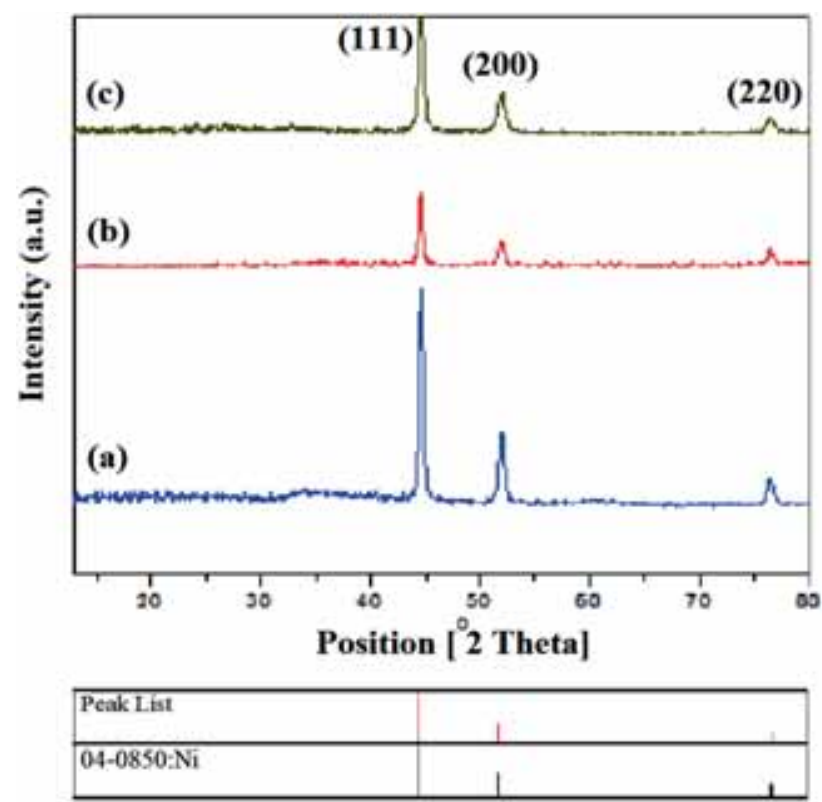

Figure 1. XRD patterns of the SNNPs obtained using $0.46 \mathrm{mmol}$ of $\mathrm{Ni}(\mathrm{acac})_{2}$ in DMF at different mole ratios of SDS:Ni(acac) $)_{2}$ : (a) $1: 1$, (b) $2: 1$ and (c) $4: 1$.

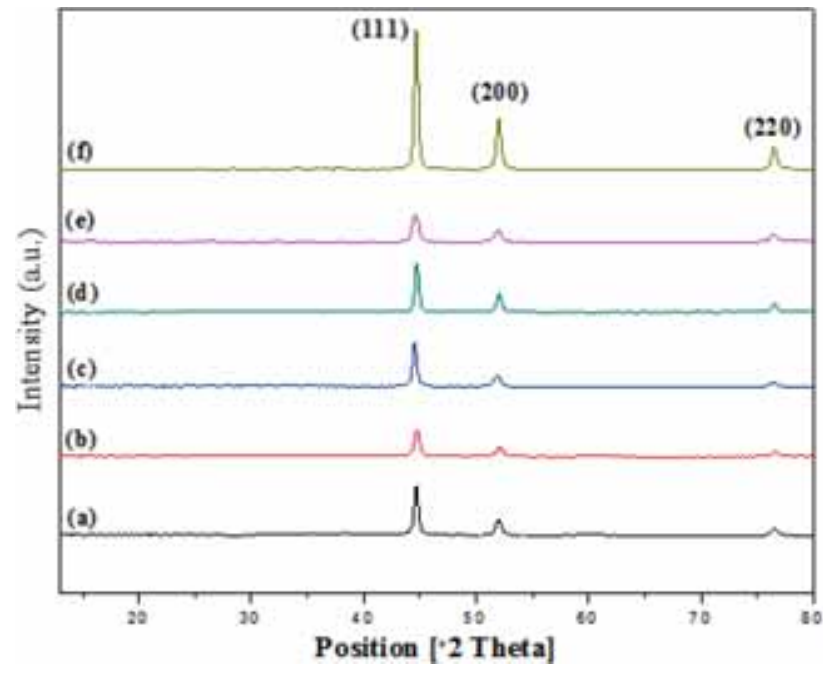

Figure 2. XRD patterns of the SNNPs obtained at $1: 1,2: 1$ and $4: 1$ mole ratios of SDS: $\mathrm{Ni}(\mathrm{acac})_{2}$ in DMF and various concentrations of $\mathrm{Ni}(\mathrm{acac})_{2}$ : (a-c) $0.70 \mathrm{mmol}$ and (d-f) $0.93 \mathrm{mmol}$.

Figure 2 presents the XRD patterns of the nickel NPs obtained using $\mathrm{Ni}(\mathrm{acac})_{2}$ of different concentrations of $(0.70$ and $0.93 \mathrm{mmol}$ ) and in the presence of various amounts of SDS. Similarly, all the reflection peaks are assigned to the crystalline $\mathrm{Ni}$ in a face-centred cubic structure (JCPDS 04-0850). The crystallite size of the as-prepared nickel nanoparticles was calculated using the Scherrer formula [27]:

$$
D=\frac{K \lambda}{\beta \cos \theta},
$$

Table 1. Crystallites size of the prepared SNNPs.

\begin{tabular}{lcc}
\hline $\begin{array}{l}\text { Ni(acac) } \\
(\mathrm{mmol})\end{array}$ & $\begin{array}{l}\text { Mole ratio of } \\
\text { SDS:Ni(acac) }\end{array}$ & $\begin{array}{l}\text { Crystallite } \\
\text { size (nm) }\end{array}$ \\
\hline 0.46 & $1: 1$ & 27.8 \\
0.46 & $2: 1$ & 25.3 \\
0.46 & $4: 1$ & 19.7 \\
0.70 & $1: 1$ & 28.0 \\
0.70 & $2: 1$ & 26.2 \\
0.70 & $4: 1$ & 24.0 \\
0.93 & $1: 1$ & 26.7 \\
0.93 & $2: 1$ & 25.1 \\
0.93 & $4: 1$ & 22.2 \\
\hline
\end{tabular}

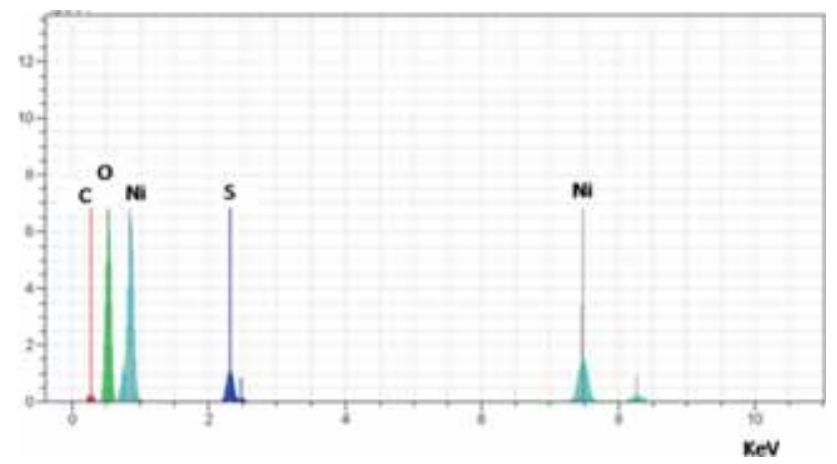

Figure 3. EDS analysis of the SNNPs obtained using $0.46 \mathrm{mmol}$ of $\mathrm{Ni}(\mathrm{acac})_{2}$ in DMF at mole ratio SDS: $\mathrm{Ni}(\mathrm{acac})_{2}=2: 1$.

where $K$ is the Scherrer constant $(K=0.89), \lambda$ is the wavelength of the $\mathrm{CuK} \alpha_{1}$ radiation $(0.1541 \mathrm{~nm}), \theta$ is the Bragg angle of the peak and $\beta$ is the corrected half-width of the diffraction peak. The average crystalline sizes of the Ni nanoparticles calculated by the Scherrer formula are presented in table 1 . The average crystallites size of the SNNPs decreased as the SDS concentration increased; the SDS molecules encapsulated the newly formed nanoparticles and prevented their further growth. On increasing the SDS concentration, an adequate amount of SDS molecules completely attached to the nanoparticles, preventing their further growth, which resulted in smaller size NPs.

EDS was used to determine the chemical composition of nickel NPs (figure 3). EDS analysis on SNNP obtained using $0.46 \mathrm{mmol}$ of $\mathrm{Ni}(\mathrm{acac})_{2}$ in DMF in the presence of SDS at mole ratio 2 showed the existence of $\mathrm{Ni}, \mathrm{S}, \mathrm{O}$ and $\mathrm{C}$. Sulphur, oxygen and carbon peaks are due to SDS adsorbed on the nanoparticles.

The FT-IR spectrum of the obtained SNNPs using SDS at the mole ratio of $\mathrm{SDS}: \mathrm{Ni}(\mathrm{acac})_{2}=2: 1$, is shown in figure 4 ; it contains a broad band at $3432 \mathrm{~cm}^{-1}$ and a band at $1629 \mathrm{~cm}^{-1}$, which are assigned to stretching and bending vibrations of adsorbed water molecules, respectively [28]. Two characteristic absorption bands are exhibited, indicative of the presence 


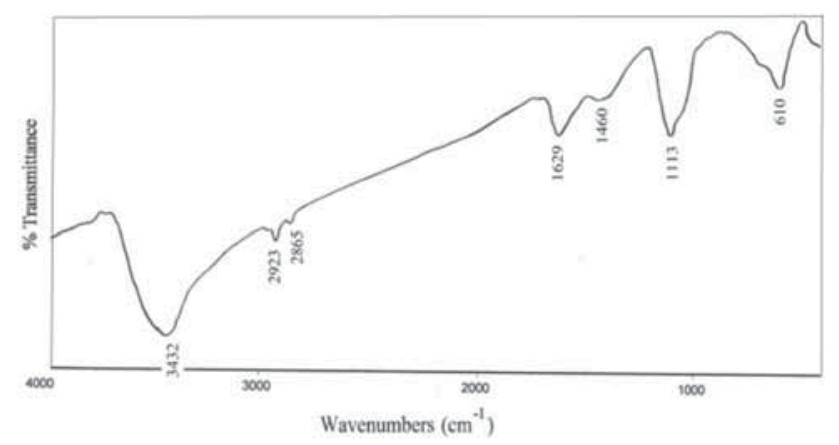

Figure 4. FT-IR spectrum of the SNNPs obtained using $0.46 \mathrm{mmol}$ of $\mathrm{Ni}(\mathrm{acac})_{2}$ in $\mathrm{DMF}$ at mole ratio SDS: $\mathrm{Ni}(\mathrm{acac})_{2}=2: 1$ using $\mathrm{KBr}$ pellets.

of sulphate groups at 1113 and $610 \mathrm{~cm}^{-1}$, respectively, corresponding to skeletal $\mathrm{S}-\mathrm{O}$ and $\mathrm{S}-\mathrm{O}-\mathrm{S}$ vibrations [29]. The C-H stretching vibrations of SDS appear at 2923 and $2865 \mathrm{~cm}^{-1}$. In addition, a weak broad band at $1460 \mathrm{~cm}^{-1}$ could be ascribed to the $\mathrm{C}-\mathrm{O}$ stretching vibration of SDS arrangement. These evidences indicate the existence of SDS adsorbed on the Ni NPs.

The morphology of magnetic SNNPs was characterized using the SEM. Figure 5a shows the SEM image of the obtained Ni NPs using $0.46 \mathrm{mmol}$ of $\mathrm{Ni}(\mathrm{acac})_{2}$ in DMF at $150^{\circ} \mathrm{C}$ for $24 \mathrm{~h}$ by introducing SDS at the mole ratio of SDS: Ni(acac $)_{2}=1: 1$. The product is sphere-shaped but the homogeneity of the morphology is low. The corresponding histogram of the particles size distribution is shown in figure $5 \mathrm{~A}$. The dominant particle size is $30 \mathrm{~nm}$, which was calculated using Digimizer software. As can be observed in figure $5 \mathrm{~b}$, on incrementing the mole ratio of SDS: $\mathrm{Ni}(\mathrm{acac})_{2}$ to 2:1, different locations of the Ni NPs were homo-dispersed. The obtained nanoparticles were uniform and the average particles size was found to be approximately $20 \mathrm{~nm}$ (figure 5B). On rising the mole ratio of SDS: $\mathrm{Ni}(\mathrm{acac})_{2}$ to $4: 1$, the size of the Ni particles decreased to $18 \mathrm{~nm}$ as shown in figure $5 \mathrm{C}$. Surprisingly, the small particles tend to coalesce and agglomerate, which results in non-uniform quasi-spherical particles (figure 5c). Apparently, these particles are composed of fine nanoparticles interconnecting the big ones. Thus, the size and distribution of the formed nanoparticles strongly depend on the stabilizer concentration.

TEM image of the obtained Ni NPs using $0.46 \mathrm{mmol}$ of $\mathrm{Ni}(\mathrm{acac})_{2}$ in the presence of SDS at the mole ratio of SDS: $\mathrm{Ni}(\mathrm{acac})_{2}=2: 1$ is given in figure 6 . It exhibits the presence of homogenously dispersed, spherical and uniform particles. As the figure depicts, the average particle size is about $15 \mathrm{~nm}$.

To determine the effect of precursor concentration on nucleation and growth of nickel NPs, the reactions were carried out at various concentrations of $\mathrm{Ni}(\mathrm{acac})_{2}(0.70$ and 0.93 $\mathrm{mmol}$ ), in DMF solvent at $150^{\circ} \mathrm{C}$ for $24 \mathrm{~h}$. Figure $7 \mathrm{a}, \mathrm{b}$ and $\mathrm{c}$ shows the SEM images of the as-prepared Ni NPs obtained using $0.70 \mathrm{mmol}$ of $\mathrm{Ni}(\mathrm{acac})_{2}$ and SDS at the mole ratios of SDS:Ni(acac $)_{2}=1: 1,2: 1$ and 4:1, respectively. As can be seen in figure $7 \mathrm{a}$ and $\mathrm{b}$, it is observed that the quasi-spherical particles are almost non-uniform in size and the average particles size was 38 and $34 \mathrm{~nm}$, respectively, as presented in figure 7A and B. Rising the mole ratio of SDS: $\mathrm{Ni}(\mathrm{acac})_{2}$ to 4:1 yielded porous sphere-shaped particles composed of small
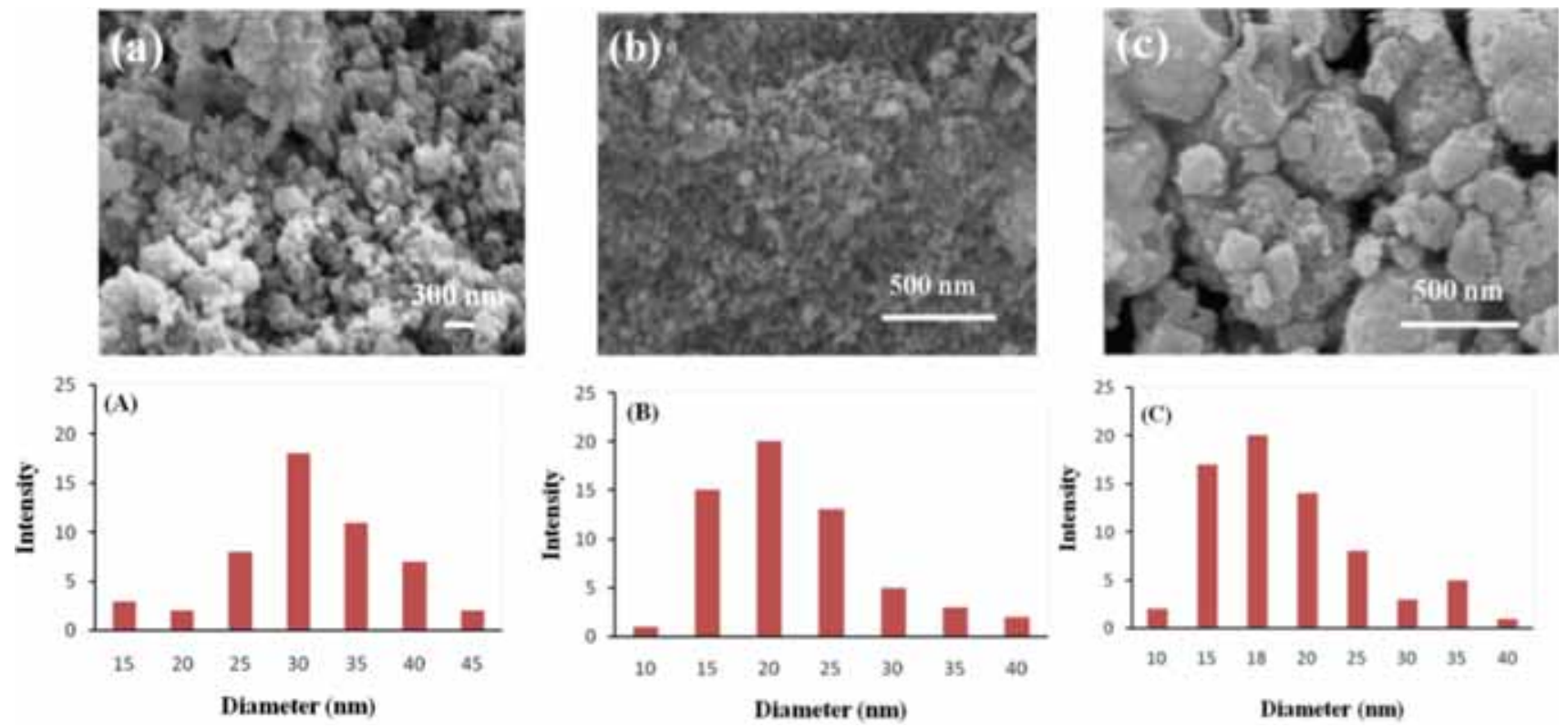

Figure 5. SEM images of the SNNPs obtained using $0.46 \mathrm{mmol}$ of $\mathrm{Ni}(\mathrm{acac})_{2}$ in DMF at different mole ratios of SDS:Ni(acac) $)_{2}$ : (a) $1: 1$ and (b) 2:1 (inset shows particle size distribution of SNNPs) and (c) 4:1. The corresponding histograms of the particle size distribution are shown in $\mathbf{A}-\mathbf{C}$. 


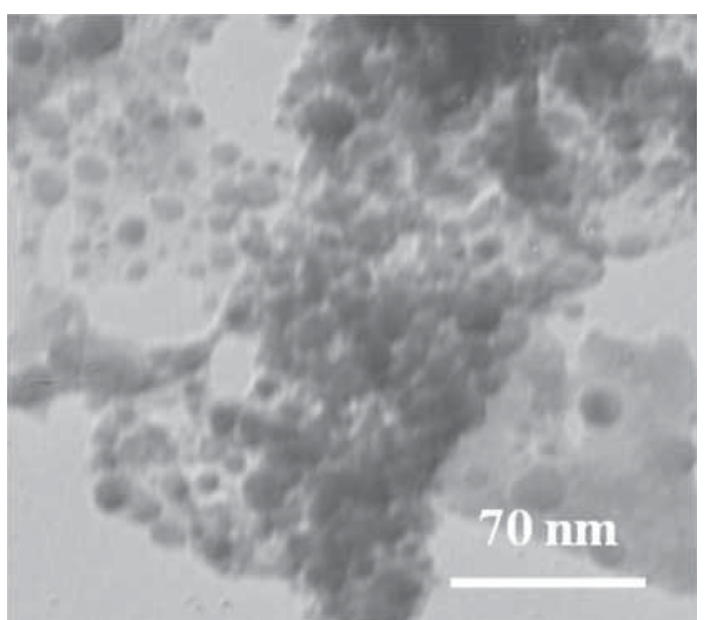

Figure 6. TEM image of the SNNPs obtained using $0.46 \mathrm{mmol}$ of $\mathrm{Ni}(\text { acac })_{2}$ in DMF at 2:1 mole ratio of SDS:Ni(acac) $)_{2}$.

particles (32 nm) as shown in figure $7 \mathrm{c}$ and C). As mentioned earlier, it could be deduced that the small particles coalesced together and big ones were created on increasing the concentration of SDS in our reaction conditions.

Particularly, the morphology of SNNPs is altered on rising the amount of $\mathrm{Ni}(\mathrm{acac})_{2}$ to $0.96 \mathrm{mmol}$ using SDS in the mole ratio 1 and keeping other reaction parameters unaltered (figure 7d). The prepared nanostructures consist of sheet-like particles that are located next to each other both horizontally and vertically, which appear in twisted and spiral shape with an average particle size of $26 \mathrm{~nm}$ (figure 7D). Figure 7e presents SEM image showing the features of the SNNPs synthesized by adding SDS at the mole ratio 2 with other conditions being constant. As can be seen in figure 7e, the small nanoparticles seem to aggregate and cause formation of big sphere-shaped particles, which are composed of small nanoparticles with an average particle size of nearly $24 \mathrm{~nm}$ (figure 7E). It was noted that, the nanosheets were inclined to coalesce and then switched to sphere-shaped particles on increasing the amount of stabilizer. Figure $7 \mathrm{f}$ shows that at high content of SDS at the mole ratio 4, the biggest sphereshaped particles having very fine particles of about $22 \mathrm{~nm}$ size are formed. The related histogram of the particles size distribution is presented in figure 7F. The obtained results show that the variations in the concentrations of $\mathrm{Ni}(\mathrm{acac})_{2}$ and SDS are seemingly associated with the variation in the morphology and size of the particles.

In our previous work, we presented that DMF could act as a unique reducing agent for the reduction of $\mathrm{Ni}^{2+}$ under suitable conditions. Accordingly, the redox reaction during the heating process can be formulated as follows $[26,30]$ :

$$
\mathrm{Ni}^{2+}+\mathrm{HCONMe}_{2}+\mathrm{H}_{2} \mathrm{O} \longleftrightarrow \mathrm{Ni}+\mathrm{Me}_{2} \mathrm{NCOOH}+2 \mathrm{H}^{+}
$$$$
\mathrm{Me}_{2} \mathrm{NCOOH} \longleftrightarrow \mathrm{CO}_{2}+\mathrm{Me}_{2} \mathrm{NH}
$$

After the SDS was added as a stabilizing agent, nickel NPs were capped with the stabilizer by interaction with the $\mathrm{SO}_{4}^{2-}$ polar group in SDS arrangement and $\mathrm{Ni}^{2+}$ ions were obtained, with the nanoparticle surface. This created a protective layer around the NPs, which could promote shape control on the growth. The evidences indicate that in the SNNPs prepared at a mole ratio of SDS: $\mathrm{Ni}(\mathrm{acac})_{2}=4: 1$, self-assemblies are structured by attachment of many smaller primary nanoparticles and formation of big ones with a semispherical shape. This self-organization could be attributed to the reduced attachment rates and weak adsorption of the SDS on the surface of the particles at such stabilizer concentration, because the nanoparticles growth is controlled by the dispersion and the attachment rates of stabilizer on the nanoparticles surface. Based on the characterization results from conditiondependent experiments, we proposed a mechanism for the growth of SNNPs as displayed in scheme 1.

The study of CV for the synthesized SNNPs electrodes using $0.46 \mathrm{mmol}$ of $\mathrm{Ni}(\mathrm{acac})_{2}$ in DMF by introducing SDS at different concentrations as a stabilizer was performed to understand the electrochemical behaviour of these nanoparticles. The $\mathrm{CV}$ curves were measured using $1 \mathrm{M} \mathrm{NaOH}$ in a potential range from -1 to $0.7 \mathrm{~V}$ at a scan rate of $0.1 \mathrm{~V} \mathrm{~s}^{-1}$, which are presented in figure 8 . As can be seen in figure 8 , a redox system consisting of an oxidation peak at about $0.53 \mathrm{~V}$ of $\mathrm{Ag}-\mathrm{AgCl}$ and two reduction peaks close to 0.28 and -0.55 $\mathrm{V}$ of $\mathrm{Ag}-\mathrm{AgCl}$ were observed. The oxidation and reduction peaks are related to the redox reaction of $\mathrm{Ni}$ to $\mathrm{Ni}(\mathrm{OH})_{2}$ and $\mathrm{Ni}(\mathrm{OH})_{2}$ to $\mathrm{NiOOH}$ and vice versa in accordance with the equations (3) and (4) [31-33]:

$$
\begin{aligned}
& \mathrm{Ni}+2 \mathrm{OH}^{-} \longleftrightarrow \mathrm{Ni}(\mathrm{OH})_{2}+2 \mathrm{e}^{-} \\
& \mathrm{Ni}(\mathrm{OH})_{2}+\mathrm{OH}^{-} \longleftrightarrow \mathrm{NiOOH}+\mathrm{H}_{2} \mathrm{O}+\mathrm{e}^{-}
\end{aligned}
$$

It is notable that oxidation of $\mathrm{Ni}$ to $\mathrm{Ni}(\mathrm{OH})_{2}$ produces a peak at about $-0.5 \mathrm{~V}$ [31], while this peak seems to be missing in our CV study. The reason for this change may be is the transience of $\mathrm{Ni}(\mathrm{OH})_{2}$ and direct oxidation of $\mathrm{Ni}$ to $\mathrm{NiOOH}$ under the current reaction conditions, which created a peak at $0.53 \mathrm{~V}$. When the mole ratio of SDS: $\mathrm{Ni}(\mathrm{acac})_{2}$ is $2: 1$, the potentials of the redox peaks are increased and the reduction peaks are shifted to positive values. Thus, at this mole ratio, the electronic transfer seems to be more than those at 1:1 and 4:1 mole ratios of SDS:Ni(acac) $)_{2}$. From figure 8 it can be concluded that the concentration of the stabilizer during the synthesis of the nanoparticles has a significant effect on the shape of the voltammograms and the electrochemical behaviour of SNNPs. The stabilizer can influence the equilibrium present on the surface of the particles, i.e., the concentration of the stabilizer does not change the reaction mechanism but affects the dispersion and can be used to adjust the textural properties of the metallic particles.

To identify the electrical conductivity of electrodes, the EIS measurements of the SNNPs electrodes prepared using $0.46 \mathrm{mmol}$ of $\mathrm{Ni}(\mathrm{acac})_{2}$ in DMF in the presence of SDS at 

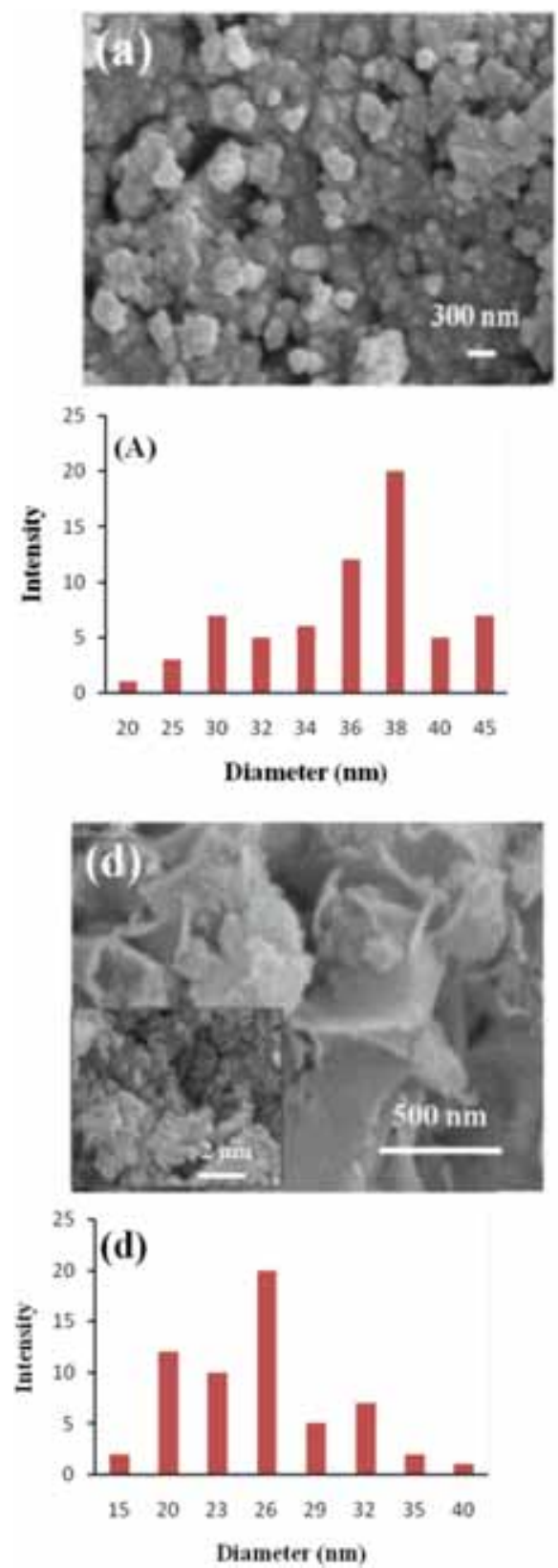
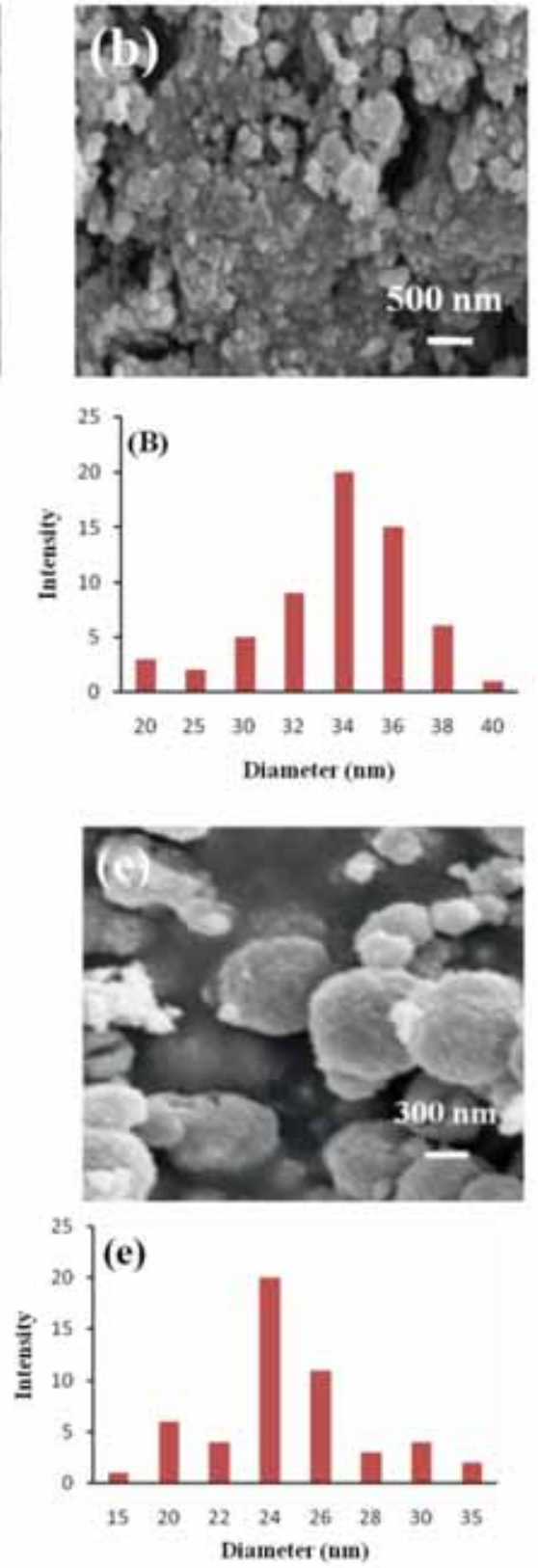
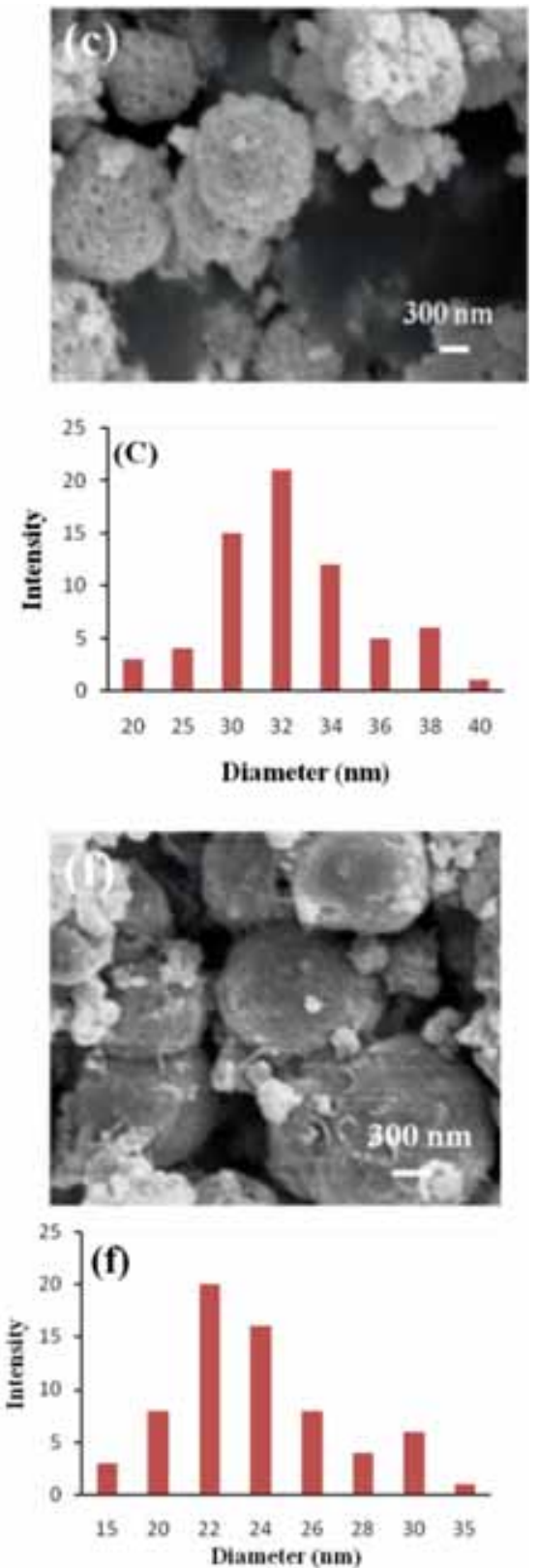

Figure 7. SEM images of the SNNPs obtained at 1:1, 2:1 and 4:1 mole ratios of SDS:Ni(acac) $)_{2}$ in DMF and various concentrations of $\mathrm{Ni}(\mathrm{acac})_{2}$ : $(\mathbf{a}-\mathbf{c}) 0.70 \mathrm{mmol}$ and $(\mathbf{d}-\mathbf{f}) 0.93 \mathrm{mmol}$. The corresponding histograms of the particle size distribution are shown in $\mathbf{A}-\mathbf{F}$.

different concentrations as the stabilizer were carried out in the frequency range $10^{5}-0.01 \mathrm{~Hz}$ in $1 \mathrm{M}$ solution of $\mathrm{NaOH}$, which are shown in figure 9a. The high-frequency regions of the spectra are shown as the inset. An equivalent circuit used to fit the impedance curve is presented in figure $9 \mathrm{~b}$, which is similar to the circuit employed for the working electrode of nickel electrodes having electrocatalytic behaviour. The EIS data can be fitted to a solution resistance $R_{\mathrm{S}}$, a Faradaic interfacial charge transfer resistance $R_{\mathrm{ct}}$ and a CPE to account for the double-layer capacitance. The charge-transfer resistance $R_{\mathrm{ct}}$ of all the samples was calculated using Nova software. From the calculated results, we found that the SNNPs electrode prepared at a mole ratio of SDS: Ni $(\mathrm{acac})_{2}=2: 1$ has the lowest value of $76.6 \Omega$. The $R_{\mathrm{ct}}$ values of electrodes at the mole ratio of SDS:Ni(acac) $)_{2}=1: 1$ and 4:1 are 187.5 and $229.8 \Omega$, respectively. This clearly demonstrates the reduced chargetransfer resistance of the electrode at these two mole ratios of SDS:Ni(acac $)_{2}$. Further, the sharp increase of the imaginary part in the Nyquist plot at lower frequency is due to the electrocatalytic activity of the electrodes towards the HER, which was modified via the concentration of the stabilizer [34]. The commonly approved mechanism for Ni electrodes in alkaline 


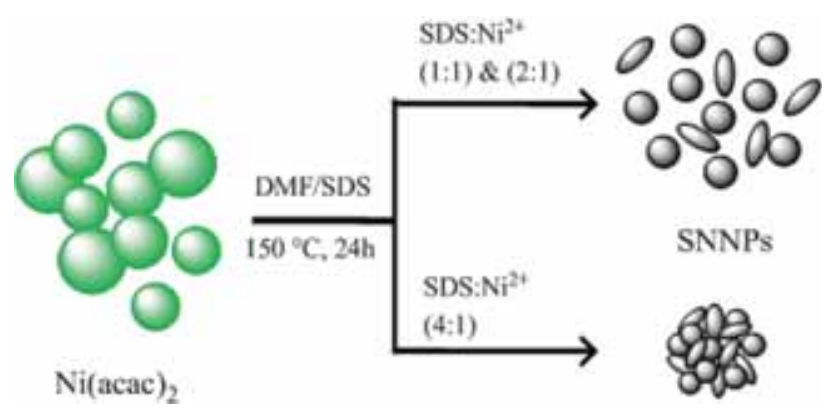

Scheme 1. Schematic diagram of the formation of SNNPs.

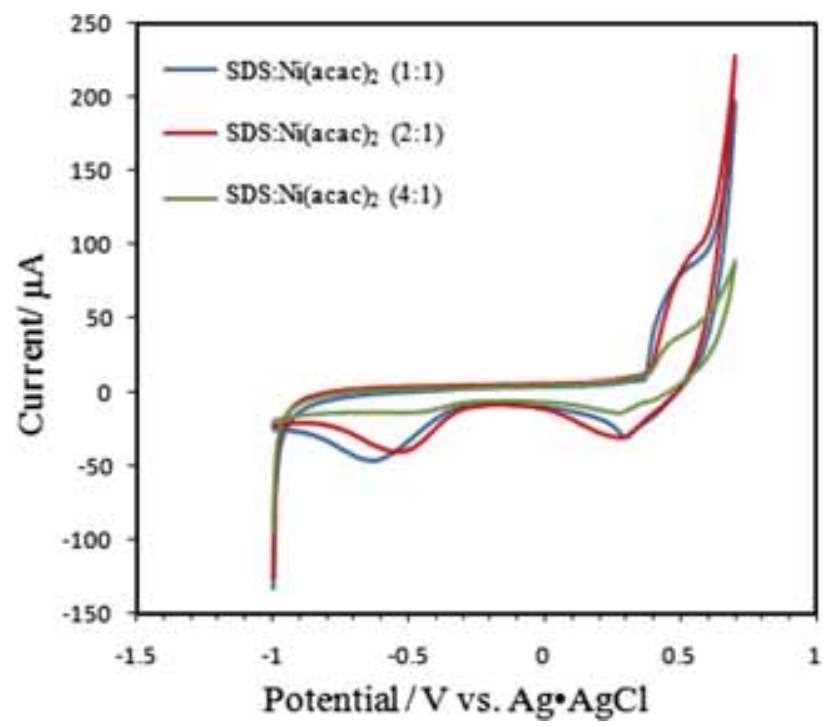

Figure 8. Cyclic voltammetries curves of the SNNPs at different concentrations of SDS, within a -1 to $0.7 \mathrm{~V}$ range at a scan rate 0.1 $\mathrm{V} \mathrm{s}^{-1}$ in $1 \mathrm{M} \mathrm{NaOH}$ electrolyte at room temperature. solutions for the HER is given in equations (5)-(7):

$$
\mathrm{Ni}+\mathrm{H}_{2} \mathrm{O}+\mathrm{e}^{-} \longleftrightarrow \mathrm{Ni}-\mathrm{H}_{\mathrm{ads}}+\mathrm{OH}^{-}
$$

Followed by

$$
\begin{aligned}
& 2 \mathrm{Ni}-\mathrm{H}_{\mathrm{ads}} \longleftrightarrow \mathrm{H}_{2}+2 \mathrm{Ni} \text { (recombination) } \\
& \mathrm{Ni}-\mathrm{H}_{\mathrm{ads}}-\mathrm{H}_{2} \mathrm{O}+\mathrm{e}^{-} \longleftrightarrow \mathrm{H}_{2}+\mathrm{OH}^{-}+\mathrm{Ni}
\end{aligned}
$$

(electrochemical desorption)

This part of the spectra was ascribed to the faradic impedance and the low-frequency resistance dominates over a broad region of low frequency. Moreover, the electrocatalytic activity of the stabilized Ni nanoparticles electrode was found to be high when the SDS: $\mathrm{Ni}(\mathrm{acac})_{2}$ mole ratio was $2: 1$ because the electron transfer rate between the SNNPs electrode materials and the charge collector is large.

The magnetic hysteresis loop of the synthesized SNNPs using $0.46 \mathrm{mmol}$ of $\mathrm{Ni}(\mathrm{acac})_{2}$ in DMF in the presence of SDS at mole ratio $\mathrm{SDS}: \mathrm{Ni}(\mathrm{acac})_{2}=2: 1$ measured at $25^{\circ} \mathrm{C}$ is shown in figure 10. It exhibits the typical ferromagnetic behaviour of metallic Ni. Magnetic properties calculated from $M-H$ loop are given in table 2. For comparison, magnetic properties of the nickel NPs prepared without any stabilizer, which have been measured previously [26], and bulk nickel are presented in table 2. Although the bulk nickel has coercivity of about 100 Oe $[35,36]$, the nickel NPs obtained using SDS exhibit considerably enhanced coercivity (185 Oe), even higher than that of the nickel NPs obtained in the absence of SDS under the same reaction conditions (175 Oe) [26]. The higher coercivity of the nickel nanoparticles obtained using SDS is possibly due to the relatively higher anisotropy of the crystallites particles [37]. It is recognizable from figure 10 and table 2 that the synthesized nickel NPs using SDS have a relatively low
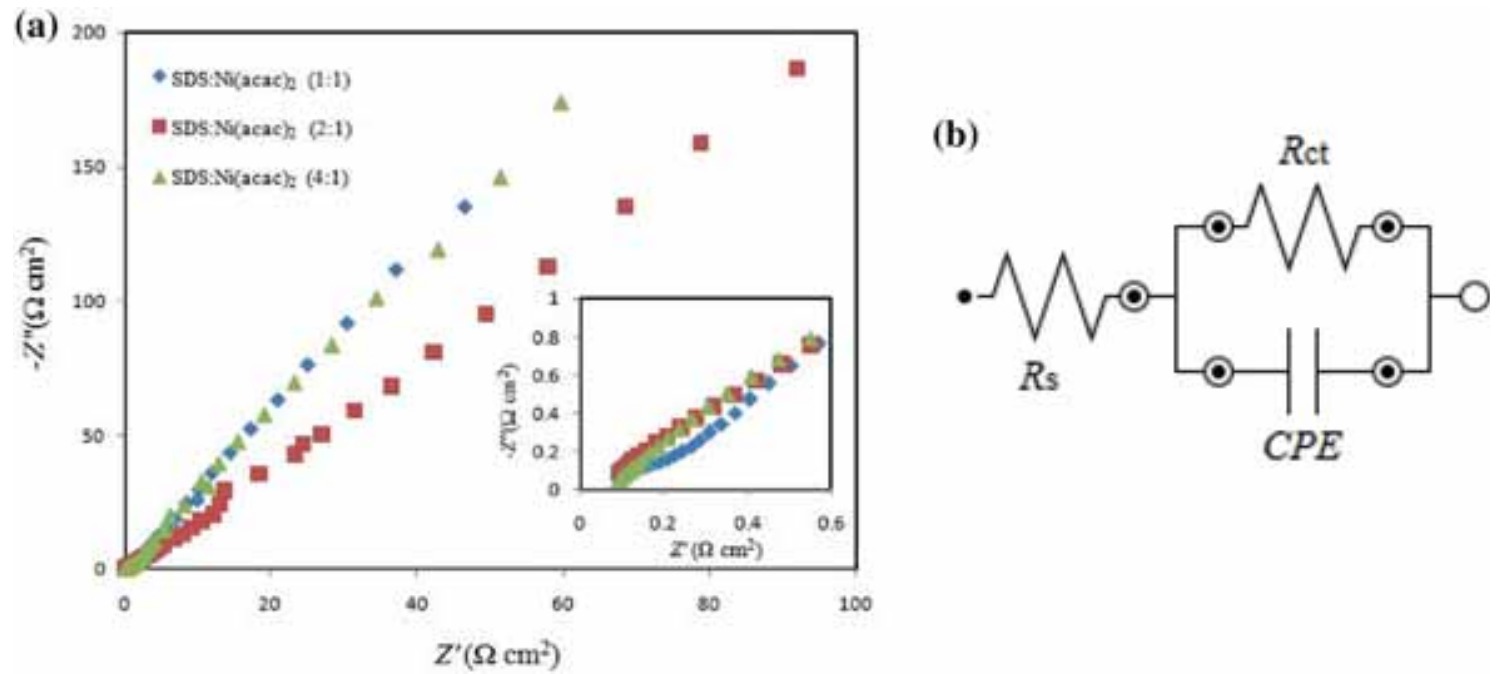

Figure 9. (a) Nyquist plots of the impedance of the SNNPs at an overpotential of $-400 \mathrm{mV}$ in $1 \mathrm{M} \mathrm{NaOH}$ electrolyte at room temperature. (b) Equivalent circuit of the SNNPs for the impedance spectra. 


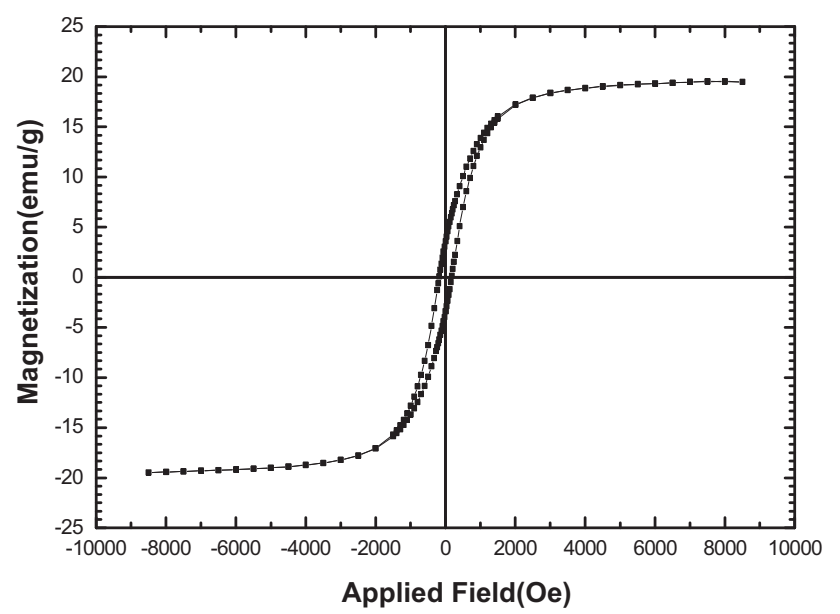

Figure 10. Room temperature magnetization curve of hysteresis loop of SNNPs obtained using $0.46 \mathrm{mmol}$ of Ni(acac $)_{2}$ in DMF at mole ratio SDS: $\mathrm{Ni}(\mathrm{acac})_{2}=2: 1$.

Table 2. Magnetic properties obtained from $M-H$ loops.

\begin{tabular}{lccc}
\hline & & $\begin{array}{l}\text { Saturation } \\
\text { magnetiza- } \\
\text { tion }\left(M_{\mathrm{S}},\right.\end{array}$ & $\begin{array}{l}\text { Remanent } \\
\text { magnetiza- } \\
\text { tion }\left(M_{\mathrm{r}},\right. \\
\left.\mathrm{emu} \mathrm{g} \mathrm{g}^{-1}\right)\end{array}$ \\
$\begin{array}{c}\text { Coercivity } \\
H_{\mathrm{c}}(\mathrm{Oe})\end{array}$ & 19 & 3.57 \\
\hline $\begin{array}{l}\text { Ni NPs obtained } \\
\text { in the presence } \\
\text { of SDS }\end{array}$ & 185 & & \\
$\begin{array}{l}\text { Ni NPs obtained } \\
\text { in the absence } \\
\text { of SDS }\end{array}$ & 175 & 42 & 9.3 \\
\begin{tabular}{l} 
Bulk Ni \\
\hline
\end{tabular} & 100 & 55 & 2.7 \\
\hline
\end{tabular}

saturation magnetization $\left(M_{\mathrm{s}}\right)$ and remanent magnetization $\left(M_{\mathrm{r}}\right)$ compared with nickel NPs without any stabilizer. These changes may be attributed to reduction in particles size and thus increase in surface area [38]. Mainly, it was due to the presence of disordered structure on particles surface and the existence of SDS as a stabilizer on the nickel NPs.

\section{Conclusions}

Although many of the previous works that show a dramatic effect on modifying the morphology of Ni nanoparticles focus on the choice of stabilizers, their reaction conditions and processes are complicated. In this work, SNNPs were prepared by thermal reduction of $\mathrm{Ni}(\mathrm{acac})_{2}$ by introducing SDS as a stabilizing agent via a simple solvothermal method. The resultant nanoparticles have been characterized as pure fcc cubic nickel by XRD. It was found that increasing the SDS concentration (mole ratios of SDS: $\mathrm{Ni}(\mathrm{acac})_{2}=1: 1,2: 1$ and 4:1) led to decrease in average crystallites size of the SNNPs. Particularly, at the 4:1 mole ratio of SDS:Ni(acac $)_{2}$, the small particles tend to merge and form big ones. It is shown that the concentration of the stabilizer exerts a strong influence on electrochemical properties of the samples as revealed by the CV and EIS measurements. It is noteworthy that the higher faradaic current density and reduced faraday resistance observed in EIS measurements support the higher electrocatalytic performance obtained in the nickel electrode when the SDS:Ni(acac) ${ }_{2}$ mole ratio is 2:1. Hence, these SNNPs could be used as electrode materials for HER. It can be concluded that the use of SDS as the stabilizer in a thermal reduction approach presented here has proved to be a useful and practical choice to obtain homogenously dispersed sphereshaped SNNPs having special electrochemical and magnetic properties.

\section{Acknowledgements}

We are grateful to University of Kashan for supporting this work by Grant Number 159183/34.

\section{References}

[1] Kisker H, Gessmann T, Wurschum R, Kronmuller H and Schaefer H E 1995 Nanostruct. Mater. 6925

[2] Aus M J, Szpunar B, El-Sherik A M, Erb U, Palumbo G and Aust K T 1992 Scr. Metal. Mater. 271639

[3] David B, Pizurova N, Schneeweiss O, Bezdička P, Morjan I and Alexandrescu R 2004 J. Alloys Compd. 378112

[4] Broto J M, Ousset J C, Rakoto H, Askenazy S, DuforCh, Brieu M and Mauret P 1993 Solid State Commun. 85263

[5] Tang N J, Zhong W, Jiang H Y, Han Z D, Zou W Q and Du Y W 2004 Solid State Commun. 13271

[6] Bai A and Hu C C 2003 Electrochem. Commun. 578

[7] Park S J, Kim S, Lee S, Khim Z G, Char K and Hyeon T 2000 J. Am. Soc. 1228581

[8] Zhang W X, Wang C B and Lien H L 1998 Catal. Today 40 387

[9] Hernandez R, Polizu S, Turenne S and Yahia L 2002 Bio. Med. Mater. Eng. 1237

[10] Krstajic N V, Jovic V D, Gajic-Krstajic L J, Jovic B M, Antozzi A L and Martelli G N 2008 Int. J. Hydrogen Energy 333676

[11] Kellenberger A, Vaszilcsin N, Brandl W and Duteanu N 2007 Int. J. Hydrogen Energy 323258

[12] Dominguez-Crespo M A, Ramirez-Meneses E, Montiel-Palma V, Torres Huerta A M and Dorantes Rosales H 2009 Int. J. Hydrogen Energy 341664

[13] Alagiri M, Muthamizhchelvan C and Ponnusamy S 2011 Mater. Lett. 651565

[14] Bao J, Liang Y, Xu Z and Si L 2003 Adv. Mater. 151832

[15] Zhang H T, Wu G, Chen X H and Qiu X G 2006 Mater. Res. Bull. 41495

[16] Yang J, Feng B, Liu Y, Zhang Y, Yang L, Wanga Y et al 2008 Appl. Surf. Sci. 2547155

[17] Altınçekiç T G, Boz I, Basaran A C, Akta B and Kazan S 2012 J. Supercond. Nov. Magn. 252771

[18] Degen A and Macek J 1999 Nanostruct. Mater. 12225

[19] De Caro D and Bradley J S 1997 Langmuir 133067 
[20] Couto G G, Klein J J, Schreiner W H, Mosca D H, de Oliveira A J A and Zarbin A J G 2007 J. Colloid Interface Sci. 311 461

[21] Hou Y, Kondoh H, Ohta T and Gao S 2005 Appl. Surf. Sci. 241 218

[22] Liu Z, Li S H, Yang Y, Peng S H, Hu Z and Qian Y $2003 A d v$. Mater. 151946

[23] Chou K S and Huang K Ch 2001 J. Nanopart. Res. 3 127

[24] Tang S H, Zheng Z, Vongehr S and Meng X 2011 J. Nanopart. Res. 137085

[25] Sarkar S, Sinha A K, Pradhan M, Basu M, Negishi Y and Pal T 2011 J. Phys. Chem. C 1151659

[26] Akbarzadeh R and Dehghani H 2014 Dalton Trans. 43 5474

[27] Dong W, An L, Wang X, Li B, Chen B and Tang W $2011 \mathrm{~J}$. Alloys Compd. $\mathbf{5 0 9} 2170$

[28] Jeevanandam P, Koltypin Y and Gedanken A 2001 Nano Lett. 1263
[29] Nakamoto K 1997 Infrared spectra of inorganic and coordination compounds (New York: John Wiley)

[30] Akbarzadeh R, Dehghani H and Behnoudnia F 2014 Dalton Trans. 4316745

[31] Roy P S and Bhattacharya S K 2014 RSC Adv. 413892

[32] Abdel Rahim M A, Abdel Hameed R M and Khalil M W 2004 J. Power Sources 134160

[33] Seghiouer A, Chevalet J, Barhoun A and Lantelme F $1998 \mathrm{~J}$. Electroanal. Chem. 442113

[34] Shervedani R K and Madram A R 2007 Electrochim. Acta 53 426

[35] Hwang J H, Dravid V P, Teng M H, Host J J, Elliott B R, Johnson D L and Mason T O 1997 J. Mater. Res. 121076

[36] Guo L, Liu C M, Wang R M, Xu H B, Wu Z Y and Yang S H 2003 J. Am. Chem. Soc. 1264530

[37] Jia J C, Yu J C, Wang Y J and Chan K M 2010 Appl. Mater. Interfaces 22579

[38] Shafi K V P M, Gedanken A, Prozorov R and Balogh J 1998 Chem. Mater. 103445 